\title{
Poisson Statistical Model of Ultrasound Super- Resolution Imaging Acquisition Time
}

\author{
Kirsten Christensen-Jeffries, Jemma Brown, Sevan Harput, Ge Zhang, Jiaqi Zhu, Meng-Xing Tang, \\ Chris Dunsby and Robert J. Eckersley.
}

\begin{abstract}
A number of acoustic super-resolution techniques have recently been developed to visualize microvascular structure and flow beyond the diffraction limit. A crucial aspect of all ultrasound super-resolution (SR) methods using single bubble localization is the time-efficient detection of individual bubble signals. Due to the need for bubbles to circulate through the entire vasculature during acquisition, slow flows associated with the microcirculation limit the minimum acquisition time needed to obtain adequate spatial information. Here, a model is developed to investigate the combined effects of imaging parameters, bubble signal density, and vascular flow on SR image acquisition time. We find that the estimated minimum time needed for SR increases for slower blood velocities and greater resolution improvement. To improve from a resolution of $\lambda / 10$ to $\lambda / 20$ while imaging the microvasculature structure modelled here, the estimated minimum acquisition time increases by a factor of 14 . The maximum useful image acquisition frame rate for an imaging depth of $5 \mathrm{~cm}$ is set by the bubble velocity at low blood flows $(<150 \mathrm{~mm} / \mathrm{s})$ and by the acoustic wave velocity at higher bubble velocities. Furthermore, the image acquisition procedure, transmit frequency, localization precision, and desired super-resolved image contrast together determine the optimal acquisition time achievable for a fixed flow velocity. Exploring the effects of both system parameters and details of the target vasculature can allow better choice of acquisition settings, and provide improved understanding of the completeness of SR information.
\end{abstract}

Index Terms - Biomedical imaging, Microbubbles, Microvasculature, Ultrasonic imaging, Ultrasound, Resolution, Poisson statistics.

\section{INTRODUCTION}

$\mathrm{N}$ on-invasive imaging of the microvasculature is crucial for the early detection and intervention of

This work was supported in part by the EPSRC under Grant EP/N015487/1 and EP/N014855/1, in part by King's College London and Imperial College London EPSRC Centre for Doctoral Training in Medical Imaging (EP/L015226/1), in part by the Wellcome Trust IEH Award [102431], in part by the Wellcome EPSRC Centre for Medical Engineering at King's College London (WT 203148/Z/16/Z), and in part by the Department of Health through the National Institute for Health Research comprehensive Biomedical Research Center Award to Guy's and St Thomas' NHS Foundation Trust in partnership with King's College London and King's College Hospital NHS Foundation Trust. diseases such as cancer [1], [2], ischemia [3] and peripheral arterial disease [4]-[6].

Acoustic super-resolution (SR) techniques have recently been developed to visualize microvascular structure and flow beyond the diffraction limit using microbubble contrast agents [7]-[16]. A crucial aspect of all methods based on single-bubble localization is the detection of spatially isolated signals from microbubbles [17]-[19]. Obtaining these isolated signals may be achieved in a number of ways, including the use of suitable microbubble concentrations with contrast imaging modes and background subtraction techniques [7], [8], or linear imaging techniques with singular value decomposition [11], [12], differential imaging [13], differential imaging with spatiotemporal nonlocal means filtering [14] or background subtraction methods [9][15][20]. Furthermore, the recent use of nanodroplets for US-SR has the benefit of providing sparsely activated microbubbles without the requirement for sufficient blood flow [21], [22]. In all these cases, the number of individual microbubble signals detected and localized per frame is restricted by the diffraction limited nature of the acquired data. Since these methods rely upon the combined localization information gathered over a series of frames, minimizing potentially long acquisition

K. Christensen-Jeffries, J. Brown, and R. J. Eckersley are with the Biomedical Engineering Department, Division of Imaging Sciences, Kings College London, London, UK (phone: 0207188837; e-mail: kirsten.christensen-jeffries@kcl.ac.uk and robert.eckersley@kcl.ac.uk).

C. Dunsby is with the Department of Physics Imperial College London, London, SW7 2AZ, UK and the Centre for Pathology, Imperial College London, London, W12 0NN, UK (email: christopher.dunsby@imperial.ac.uk).

S. Harput, G. Zhang, J. Zhu, and M. X. Tang are with the Department of Bioengineering, Imperial College London, London, SW7 2AZ, UK (email: s.harput@imperial.ac.uk and mengxing.tang@imperial.ac.uk). 
times is crucial.

In previous work, SR has been demonstrated using both conventional line-by-line focused acquisition with frame rates at or under $50 \mathrm{~Hz}$ [7], [8], and using high frame rate plane wave imaging [11]-[14], [23] where an entire 2D image plane can be acquired with one acoustic pulse [24].

Essential to the clinical translation of SR techniques is the ability to generate images in clinically viable acquisition times. This not only limits patient-clinician time requirements, but also reduces the unwanted effects of motion during long scan times.

A high localization rate can be achieved by a combination of factors. Firstly, an increase in frame rate allows more frequent sampling of microbubble flow through the vasculature. Due to the need for bubbles to circulate through the entire vasculature during acquisition, slow flows associated with the microcirculation limit the minimum acquisition time needed to obtain adequate spatial information. This therefore presents a limit to the benefit increased frame rates can provide on the acquisition time.

Secondly, each frame should contain a high number of spatially separable bubble signals. However, increasing the frame signal density (or injected microbubble concentration) does not necessarily increase the localization rate since overlapping or interfering signals should be rejected. In the event these are included, resulting incorrect localizations may be positioned outside of the vessel diameter, and thus are likely to degrade the final image.

The optimization of signal density in SR imaging is crucial to time-efficient image acquisition. The following model has been developed based on a simplified Poisson statistical model which aims to demonstrate and investigate the combined effects of acquisition imaging parameters on localization rate, and ultimately image acquisition time.

\section{MATERIALS AND METHODS}

\section{A. Number of INTERROGATIONS REQuiRED}

The acquisition time required to create a superresolved image is given by

$$
\text { Acquisition Time }=t_{i} \cdot N_{i}
$$

where $t_{i}$ is the time to acquire a single interrogation, and $N_{i}$ is the total number of interrogations needed to obtain sufficient microbubble localizations. Here, an interrogation is defined as the sequence of pulseechoes required to produce a single $2 \mathrm{D}$ frame or $3 \mathrm{D}$ volume.

To estimate the number of interrogations required, the likelihood of imaging spatially isolated microbubbles is required. Here, we image a cubic volume $V$ with sides of length $l_{x}, l_{y}, l_{z}$, at a maximum depth $d=l_{y}$, as illustrated in Figure 1 . The proportion of the local volume containing blood vessels is the local vascular volume fraction, equal to $V_{v}$, ranging from $0-100 \%$. Using a contrast agent

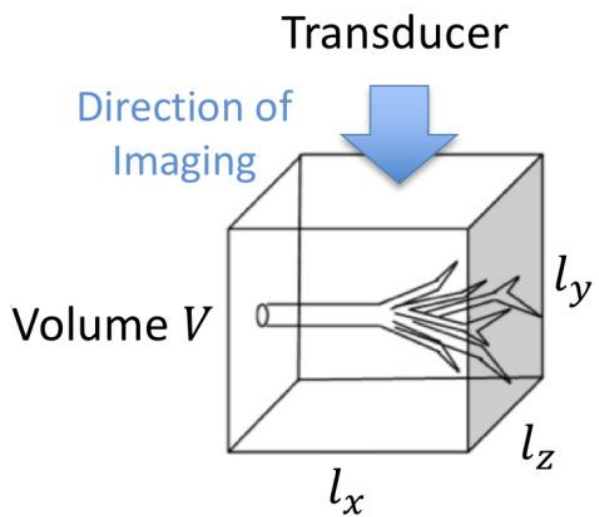

infusion, the microbubble signal density in the blood is assumed to be constant and equal to $C_{b}$.

Figure 1. Illustration of volume, $V$ imaged by the US system, where vascular structures occupy a sub-volume, defined by a variable local vascular volume fraction, $V_{v}$.

The PSF volume, or resolution voxel, $V_{\mathrm{PSF}}$, illustrated in Figure 2, can be approximated by

$$
V_{\mathrm{PSF}}=\mathrm{FWHM}_{x} \mathrm{FWHM}_{y} \mathrm{FWHM}_{z} \text {, }
$$


where $\mathrm{FWHM}_{x}$ and $\mathrm{FWHM}_{y}$ are the lateral and axial FWHM of the PSF respectively, and $\mathrm{FWHM}_{z}$ is the elevational resolution, or if acquiring $2 \mathrm{D}$ data this can be defined as the 'slice thickness', $\Delta z$. This is representative of the original diffraction limited resolution of the system. Thus, two scatterers within the same resolution voxel cannot be resolved. For simplicity, $V_{P S F}$ is represented as an isotropic voxel in diagrams, such that $\mathrm{FWHM}_{x}=\mathrm{FWHM}_{y}=$ $\mathrm{FWHM}_{z}$. In reality, the in-plane components are anisotropic, and these often differ greatly to the elevational resolution or slice thickness.

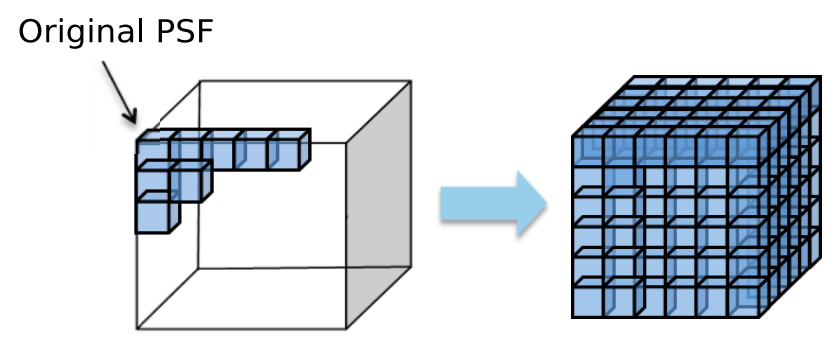

Figure 2. The imaging volume $V$ can be divided in approximately PSF sized voxels, corresponding to the three dimensional diffraction limited resolution of the imaging system.

If independent and discrete events occur with a known average rate then Poisson statistics can be used to express the probability of a given number, $k$, of events occurring within a fixed interval of time or space. In this work, an event is defined as the presence of a bubble, and the fixed spatiotemporal interval of observation is a diffraction-limited sized region within the imaging volume [25], [26]. It is assumed that bubbles do not cluster, and therefore bubble events can be defined for a finite set of values of $k$. Poisson statistics can thus be used to generate an initial distribution of bubbles from an initial expectation value [27]. We can then use this distribution to examine the probability of imaging single bubbles in a resolution voxel. In this case, the probability, $P_{P S F}$, of having $k$ bubbles in a sample volume can be given by the following relation

$$
P_{P S F}(k)=\frac{\mu^{k} e^{-\mu}}{k !}
$$

where $\mu$ is the Poisson expectation value, given by the known number of events occurring in one sample volume, $V_{P S F}$,

$$
\mu=C_{t i s} \cdot V_{P S F} .
$$

Here, $C_{t i s}$ is the concentration of bubbles in the local tissue,

$$
C_{t i s}=C_{b} \cdot V_{v}
$$

and $C_{b}$ is the microbubble signal density in the blood. Since there exists a precision associated with the localization of point scatterers using an US imaging system [7], a SR pixel, $A_{S R}$, or voxel, $V_{S R}$, can be approximated as an area or volume with sides equal in length to the localization precision in the $x, y$ and $z$ dimensions, denoted by $\sigma_{x}, \sigma_{y}$, and $\sigma_{z}$ (Figure 3), given by

$$
V_{S R}=\sigma_{x} \sigma_{y} \sigma_{z}
$$

The localization precision determines the FWHM of the Gaussian localization profile plotted for each localization in the final SR rendering as performed in our previous work in 2D [8].

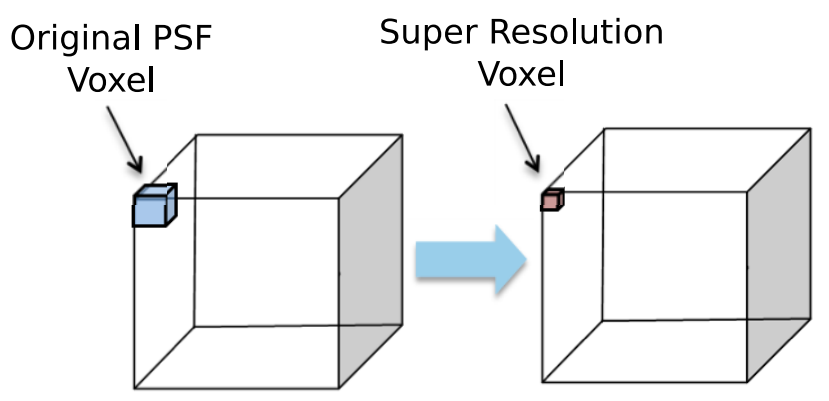

Figure 3. Illustration of the change of image resolution represented by a change in PSF voxel size, where the original resolution of the system is the PSF measured of the US imaging system, and the SR PSF is defined as a voxel size equal to the localization precision in the $x, y$ and $z$ dimensions.

The concentration of single bubble events, $C_{S B}$, is the concentration of bubbles in the tissue, multiplied by the probability that no bubbles fall into the same 
resolution cell,

$$
C_{S B}=C_{t i s} e^{-C_{t i s} \cdot V_{P S F}}
$$

The average number of single bubble detections in a super-resolution voxel, $n_{S B}$, is given by

$$
n_{S B}=C_{S B} \cdot V_{S R}
$$

The average number of localizations in a SR voxel, $N_{l}$, after $N_{i}$ interrogations is

$$
N_{l}=N_{i} C_{S B} V_{S R}
$$

This value can be chosen to define the average number of detections per SR voxel to reach a sufficient signal to noise ratio (SNR). The number of interrogations required can then be given by

$$
\begin{gathered}
N_{i}=\frac{N_{l}}{C_{S B} V_{S R}} \\
N_{i}=\frac{N_{l}}{C_{t i s} V_{S R}} \mathrm{e}^{C_{t i s} \cdot \mathrm{V}_{P S F}} .
\end{gathered}
$$

\section{B. IMAGING RATE}

The imaging rate, $I$, can be considered as the number of interrogations created per second, given by

$$
I=\frac{1}{t_{i}}
$$

During imaging, microbubbles flow through the vasculature at an average velocity $v_{b}$. For the microbubbles to provide new spatial information in each consecutive interrogation, its movement should exceed the magnitude of the system's localization precision, $\sigma=\left(\sigma_{x}, \sigma_{y}, \sigma_{z}\right)$. Since this is often not isotropic, the average localization precision over all directions is taken. Thus, the distance moved by a bubble in each interrogation, $d_{b}$, should be

$$
d_{b} \geq \operatorname{avg}(\sigma)
$$

thus

$$
v_{b} \geq \sigma \cdot I
$$

and the flow rate limit on the minimum useful time between interrogations, $t_{i F L}$, is given by

$$
t_{i F L} \geq \frac{\sigma}{v_{b}}
$$

Nevertheless, bubbles with velocity below this, or conversely, frame rates above this rate, will still contribute localizations to the final image, and thus will enhance the final SNR.

The fundamental limit on the imaging rate is determined by the time of flight and image acquisition procedure implemented. Using CEUS imaging techniques such as pulse inversion (PI) [28] or Contrast Pulse Sequencing (CPS) [29], a single line is composed of multiple pulse-echoes, $N_{\text {pulses }}$, of varying phase or amplitude. Multiple angle plane wave compounding also requires echoes from several directed wavefronts to generate each image. Thus, the minimum interrogation time, termed the acoustic limit, $t_{i A L}$, can be given by

$$
t_{i A L}=\frac{2 d N_{\text {pulses }}}{\mathrm{c}}
$$

where $c$ is the speed of sound in the medium, assumed to be constant, and $d$ is the imaging depth.

\section{Acquisition Time}

By combining relations from previous equations, an approximate overall relation can be given by

Acquisition Time

$$
=t_{i}\left(\frac{N_{l} \mathrm{e}^{C_{t i s} \cdot \mathrm{FWHM}_{x} \mathrm{FWHM}_{y} \mathrm{FWHM}_{z}}}{C_{t i s} \sigma_{x} \sigma_{y} \sigma_{z}}\right)
$$


or

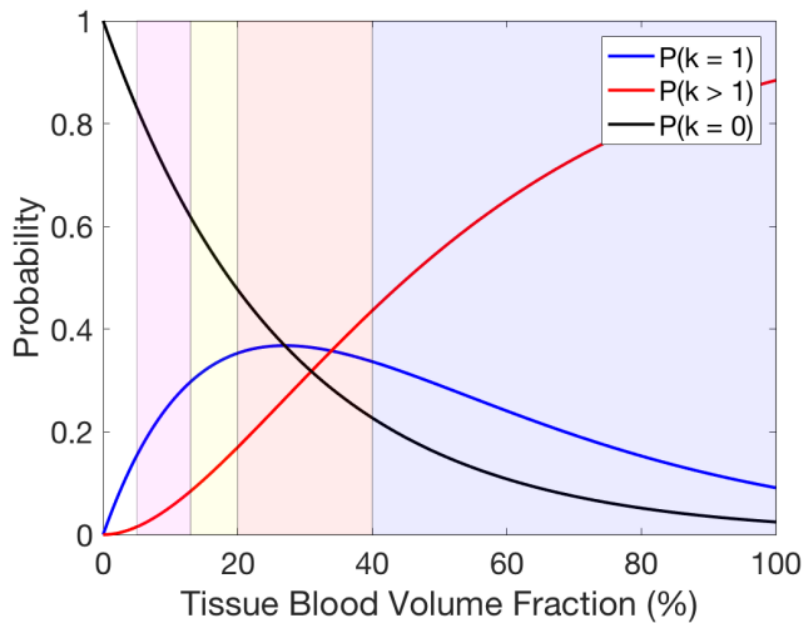

Figure 4. Probability of imaging single bubbles within the diffraction limit across tissues containing varying tissue blood volume fractions (\% of total tissue) when bubble concentration in blood is optimized for imaging the normal liver parenchyma (orange region). Ranges of blood volume fractions are also shown for regions of colangiocellular cancer (pink), hepatocellular carcinoma (yellow) and for blood volumes above $40 \%$ which may represent highly perfused tissue or macrovessels (purple). Vessels with diameter above $\lambda / 2$ are assumed to have a vascular volume fraction of $100 \%$.

$$
\text { Acquisition Time }=t_{i}\left(\frac{N_{l} \mathrm{e}^{C_{t i s} \cdot V_{P S F}}}{C_{t i s} V_{S R}}\right),
$$

where

$$
t_{i}= \begin{cases}t_{i F L}, & t_{i F L}>t_{i A L} \\ t_{i A L}, & t_{i F L} \leq t_{i A L}\end{cases}
$$

\section{INVESTIGATIONS/ SPECIFIC MODELS:}

If not varied, parameters within models are fixed under conditions typical for abdominal imaging with: transmit frequency, $4 \mathrm{MHz}$; depth, $5 \mathrm{~cm}$; flow velocity, $5 \mathrm{~mm} / \mathrm{s} ; N_{l}=1$; resolution improvement $\lambda / 30$, where $\lambda$ is the transmit wavelength, and localization precision $10 \mu \mathrm{m}$ in $2 \mathrm{D}$ where $V_{\mathrm{PSF}}=\mathrm{FWHM}_{x} \mathrm{FWHM}_{y} \Delta z$ and $A_{S R}$ is used in place of $V_{S R}$. The FWHM is estimated by $\lambda / 2$. Acquisitions were also modelled over a range of acquisition parameters, SR localization precision values, and bubble signal densities to explore the impact on acquisition time.

\section{Tissue Variations}

- Probability of Bubble Events: Infusion

The probability of imaging bubble events across varying tissue types was determined using Equations (3)-(5), where the tissue blood volume fraction ranges between that of colangiocellular cancer, hepatocellular carcinoma, normal liver parenchyma, and other highly perfused tissue or macrovessels. Models were performed using a constant microbubble infusion, where the microbubble signal density in the blood, $C_{b}$, is optimized for imaging normal liver parenchyma, i.e. where $C_{b} \cdot V_{v} \cdot V_{P S F}=1$.

\section{- Probability of Bubble Events: Bolus Injection}

The probability of imaging bubble events was determined as described above at various time-points post bolus injection. This was performed using a peak blood microbubble concentration optimized for imaging normal liver parenchyma.

\section{- Effect of Vascular Velocity}

SR acquisition times were determined using Equation (17), where bubble velocities, $v_{b}$, were varied between the slow flow of the microvasculature, up to fast flow in the aorta, using a constant microbubble infusion optimized for normal liver parenchyma.

- Effect of Transmit Frequency

The number of frames required to create a SR image with fixed abdominal parameters was modelled using transmit frequencies between 0.5-15 MHz.

\section{- Combined Effects on Acquisition Time}

SR acquisition times were again determined using Equation (17), where the combined effects of transmit frequency, signal density, level of resolution improvement, and vascular velocities were modelled.

\section{RESULTS}




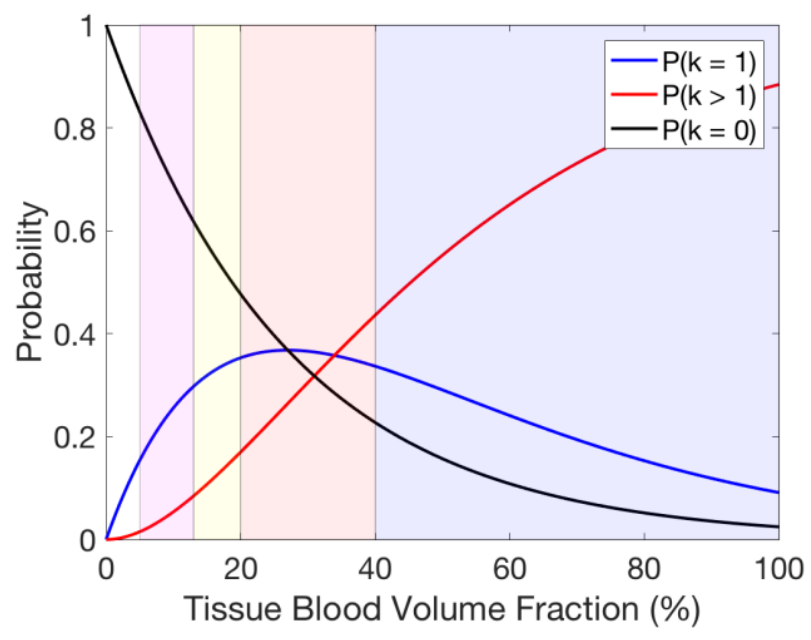

Figure 4 shows the probability of imaging bubble events within the diffraction limit across varying tissue blood volume fractions, $V_{v}$. Here, the bubble concentration in the blood is optimized for imaging normal liver parenchyma (orange region) of $26 \%$ [30] using a constant infusion. Ranges of tissue blood volumes are also shown for regions of colangiocellular cancer (pink) and hepatocellular carcinoma (yellow). Blood volumes above 40\% (purple) may represent highly perfused tissue or macrovessels. Vessels with diameter above $\lambda / 2$ are at $100 \%$ blood volume per diffraction limit. For these vessels, the probability of imaging individual

A

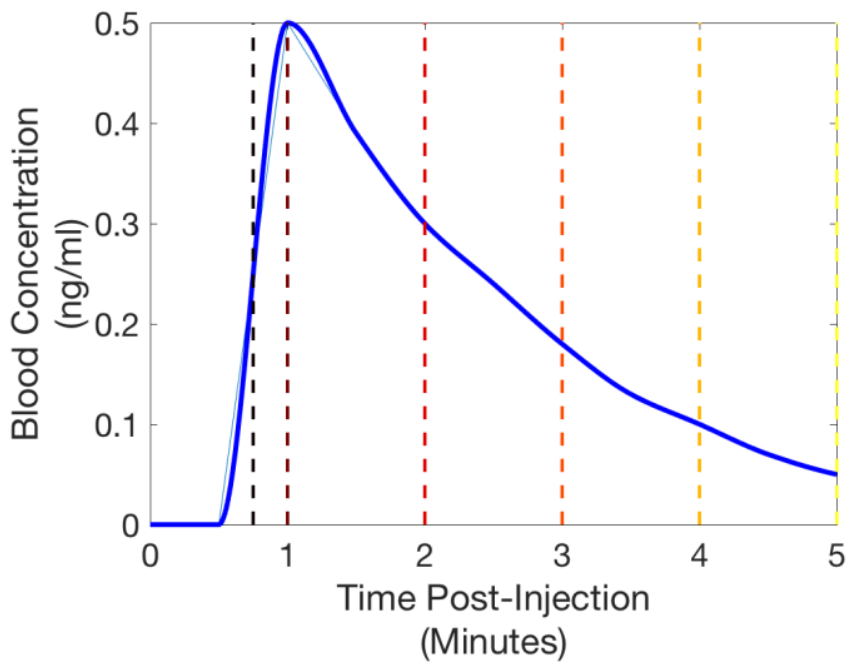

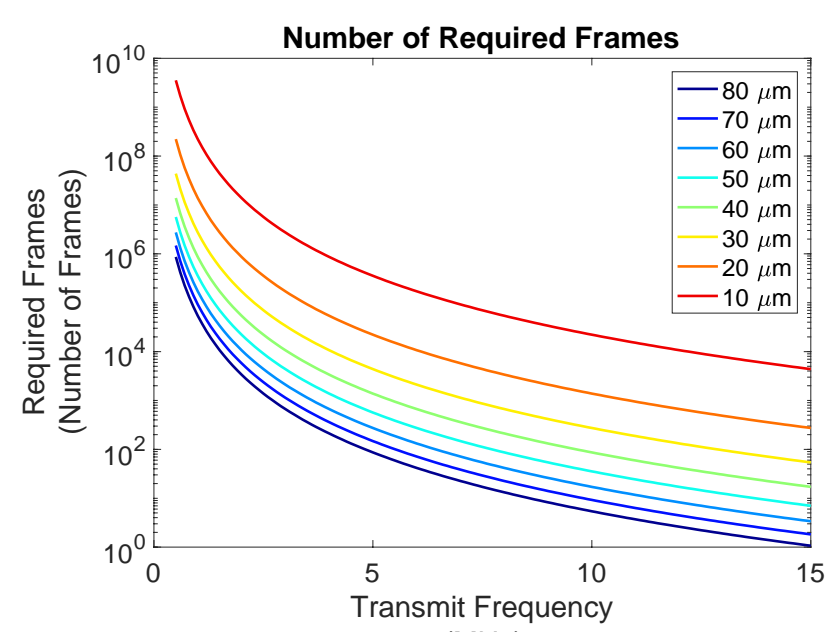

$(\mathrm{MHz})$

Figure 6. The number of frames required to create a SR image for a given localization precision, see legend. The number of frames decreases with increasing transmit frequency for fixed depth of $5 \mathrm{~cm}$ at optimum bubble concentration. Increasing the SR localization precision will increase the number of localizations required in the final image and therefore increase the number of frames needed.

bubbles at this concentration is 0.09 , while the chance of multiple bubbles occurring within the diffraction limit reaches 0.88 .

Figure 5 shows the concentration of sulphur hexafluoride in the blood following intravenous administration of SonoVue in healthy volunteers as a bolus 0.03 $\mathrm{ml} / \mathrm{kg}$ dose (Figure 5A, blue curve). This shows the probability of imaging single microbubbles within the diffraction limit for various time points postinjection (Figure 5B) demonstrated by corresponding dashed lines shown in Figure 5A. In this example, peak bubble concentration in blood is optimized for imaging cancerous tissue, i.e. for $V_{v}=13 \%$, between colangiocellular cancer (pink) and Hepatocellular Carcinoma (yellow). Ranges of blood volume

B

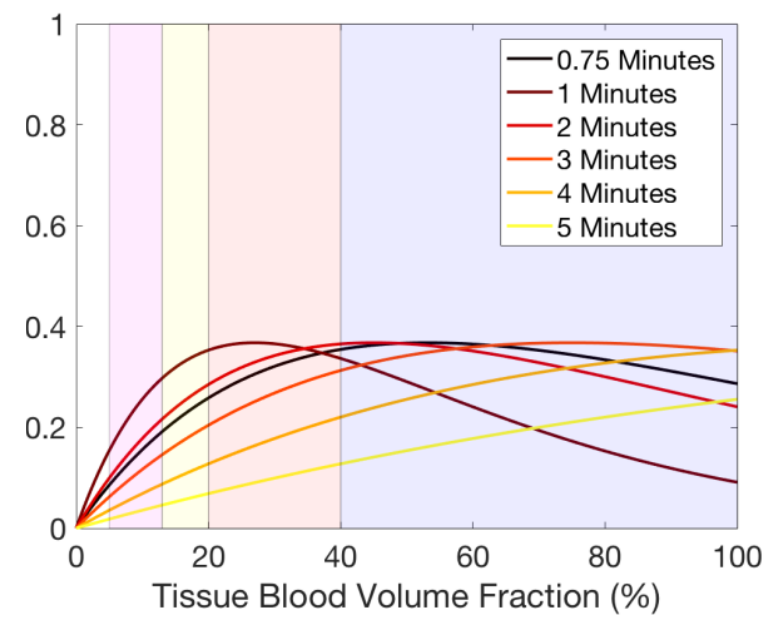

Figure 5A) Concentration of Sulphur hexafluoride in the blood following intravenous administration of SonoVue in healthy volunteers for 0.03 $\mathrm{ml} / \mathrm{kg}$ dose (thin blue curve), spline fit (thick blue curve), data taken from [37]. B) The probability of imaging single microbubbles within the diffraction limit over varying tissue tissue blood volume fractions are shown, where each curve is generated for various time points post- injection. Time points are shown by corresponding dashed lines in A). Here, peak bubble concentration in blood is optimized for imaging cancerous tissue, colangiocellular cancer (pink) and hepatocellular carcinoma (yellow). Ranges of blood volumes are also shown for regions of the normal liver parenchyma (orange region) and for blood volumes above 40\% which may represent highly perfused tissue or macrovessels (purple). 


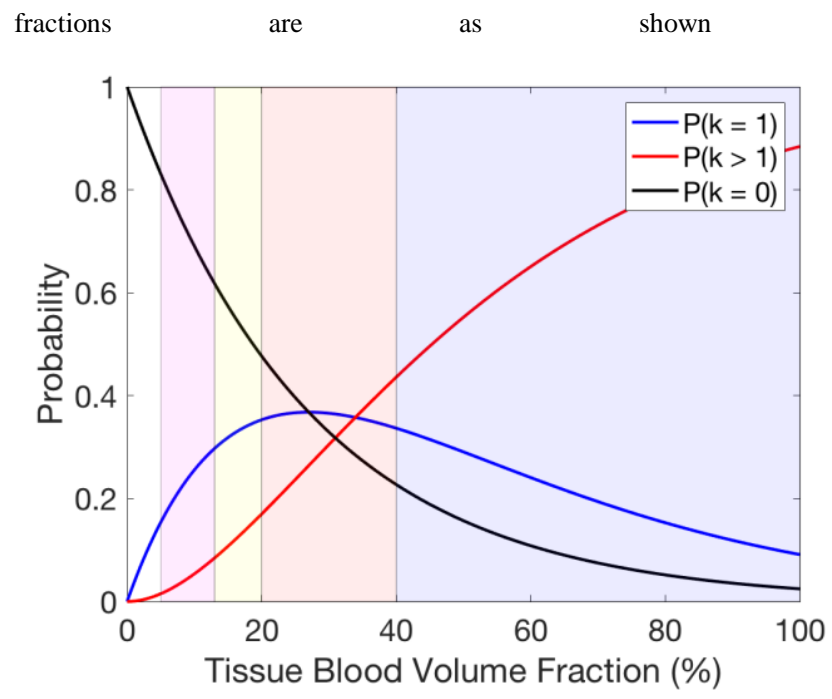

Figure 4. Figure 5B demonstrates the difference in potential detections using a bolus injection in contrast to constant infusion shown in

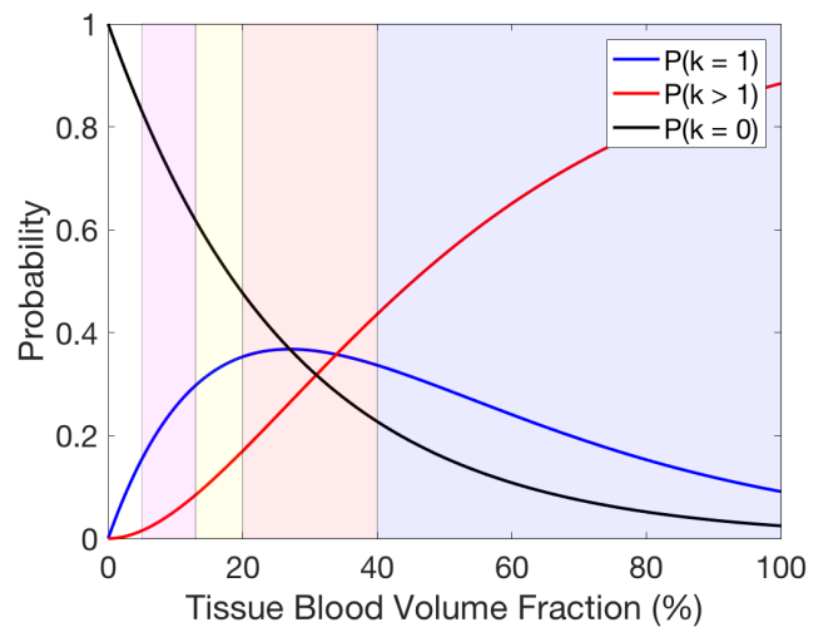

Figure 4. In this case, the optimum probability of detecting isolated bubbles occurs over a range of tissue types during bolus circulation. The steep concentration increase at inflow means optimal signal density moves from regions of high blood volume to those with lower as the blood concentration peaks. Again, at the latter part of bolus circulation, signal detections in tissues of high blood volume are likely to surpass those in less vascular regions due to the bubble concentration decrease.

The estimated number of frames required to create a SR image decreases with increasing transmit frequency (Figure 6) for a fixed depth of $5 \mathrm{~cm}$, localization precision, and at optimum bubble concentration. In general, relative improvements in the final SR precision compared with the initial diffraction limited resolution ultimately determines the number of frames required.

At $5 \mathrm{~cm}$ depth with $10 \mu \mathrm{m}$ localization precision, Figure 7A shows that the effective frame rate limits for blood velocities ranging from the microvasculature $(<1 \mathrm{~mm} / \mathrm{s})$ to the aorta $(\sim 45 \mathrm{~cm} / \mathrm{s})$ are restricted by the bubble velocity at low blood flows $(<150 \mathrm{~mm} / \mathrm{s})$, but are only restricted by the time-of-flight at higher flows. Therefore, the estimated minimum time needed for SR increases for slower blood velocities, and for improved resolution (Figure 7B). In this example, to obtain a SR of $\lambda / 10$ while imaging the microvasculature, the estimated minimum time is 56 seconds, while for $\lambda / 20$ improvement this increases to 13 minutes.

Figure 8 demonstrates the acquisition time needed to produce SR images at a fixed depth of $5 \mathrm{~cm}$ with varying bubble signal density and resolution improvement when imaging vasculature of varying flow velocities (B), resolution improvement (C), and frequencies (D and E), with fixed resolution improvement and fixed localization precision respectively. The curve shown in green shows the same conditions and is therefore identical throughout Figure 8B-E using the typical abdominal imaging parameters. The acquisition time is shown to increase considerably away from the optimum

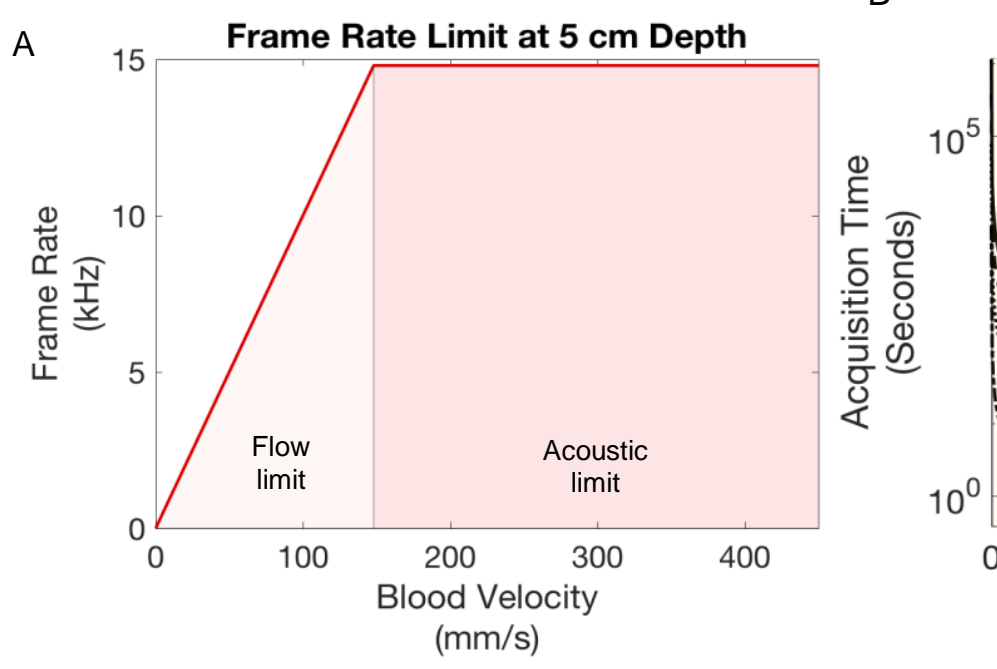

Figure 7 A) Frame rate limits are defined by the bubble velocity at low blood flows, $\mathrm{x}$ time-of-flight. Localization precision $10 \mu \mathrm{m}$. B) Acquisition time required to create sup velocities ranging between the microvasculature (yellow), veins and arterioles (red), demonstrates an increase in acquisition time with improvements in resolution compared 


\section{DISCUSSION}

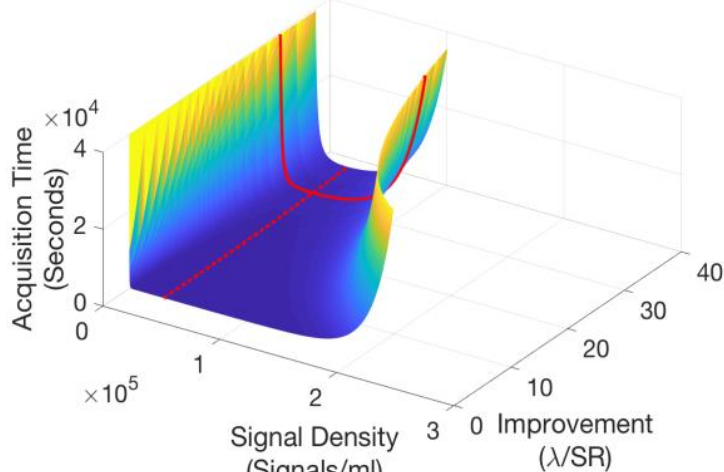

(Signals/ml)

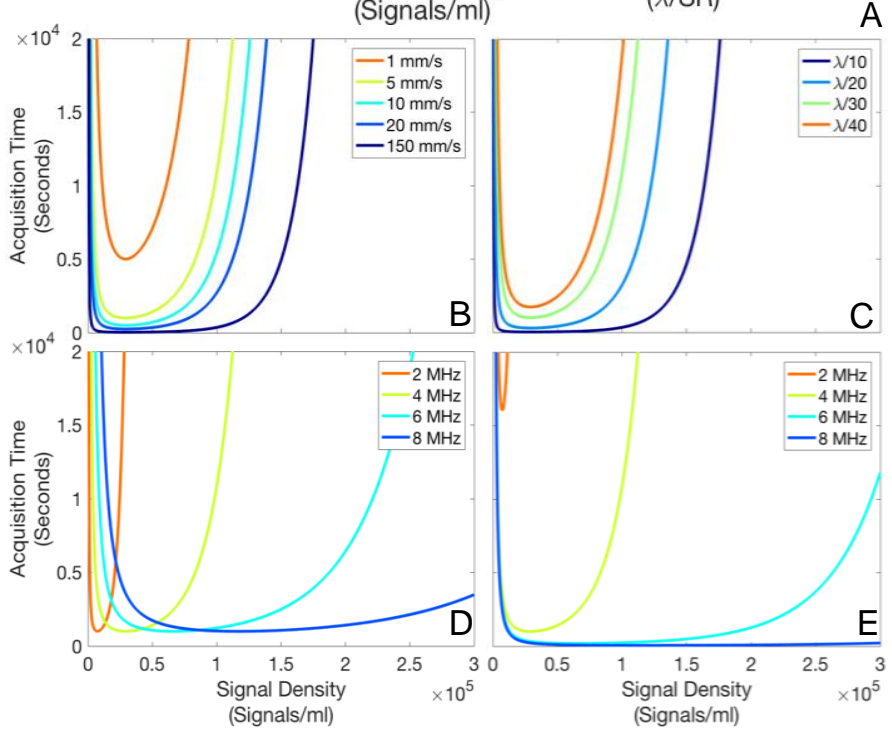

Figure 8. Acquisition time needed to produce SR images at fixed depth of $5 \mathrm{~cm}$ with varying bubble signal density and resolution improvement (A). Graphs show time required when imaging vasculature of varying flow velocities (B), resolution improvement $(\mathrm{C})$, and frequencies (D and $\mathrm{E}$ ), with fixed level of resolution improvement $(\lambda / 30)$ and fixed localization precision $(10 \mu \mathrm{m})$ respectively. Resolution improvement values for (E) are $\lambda / 62 \lambda / 31, \lambda / 21, \lambda / 15$ for $2,4,6,8 \mathrm{MHz}$ respectively. If not varied, parameters are set with transmit frequency: $4 \mathrm{MHz}$, depth: $5 \mathrm{~cm}$, flow velocity: $5 \mathrm{~mm} / \mathrm{s}$, improvement $\lambda / 30$ and localization precision: $10 \mu \mathrm{m}$. The curve shown in green is constant throughout A-D and corresponds to red line in (A). Dashed line in (A) indicates the optimum signal density.

bubble signal density, determined by the transmit frequency. Figure $8 \mathrm{~B}$ and $\mathrm{C}$ show how the minimum possible acquisition time changes with blood flow velocity and resolution improvement respectively. As the frequency lowers, the optimal signal density range narrows, however the minimum possible time at the optimum concentration remains the same for fixed resolution improvement (Figure 8D). In contrast, reducing the frequency increases the acquisition time needed to achieve the same localization precision even at optimal signal density (Figure 8E).
The acquisition procedure, transmit frequency, localization precision, bubble concentration and desired SR image contrast together determine the minimum acquisition time for a given flow velocity and tissue blood volume fraction.

This model aims to provide insight into the relationship between imaging parameters and microbubble concentration on the localization rate and overall acquisition time of SR US imaging. Under the assumptions of a Poisson distribution, this provided estimations of ideal bubble concentrations for minimizing acquisition time.

As expected, acquisition time increases with those parameters which tend to increase the acquisition time for all CEUS imaging techniques, as shown in Equation (15), including: increased imaging depth, increased number of pulses within multi-pulse CEUS imaging techniques, increased compounding angles, and a decreased speed of sound in the medium of interest. Imaging the microvasculature however, this interrogation rate becomes further limited by the slow flows imposing an effective frame rate limit. The SR component (bracketed term of Equation (17)) demonstrates that a higher diffraction limited resolution, as provided by a higher transmit frequency, acts to decrease the acquisition time. This is due to the opportunity to extract a higher number of spatially isolated bubble signals in each frame. The desired number of localizations per SR voxel, $N_{l}$, relates to the SNR in the resulting image; hence for a factor of improvement in SNR, the acquisition time required will increase by the same factor. This relies upon the algorithm's ability to separate multiple bubble events. In the event that multiple bubbles falling within one voxel are detected by the algorithm as a single detection, an incorrect localization position may be found. This may decrease the resulting SNR in the image. Thus, the importance of accurate signal detection and differentiation.

Equation (17) also demonstrates an inverse relationship between imaging time and localization 
error; this is due to the increased size of the Gaussian localization plots in the final SR image.

The results show that the use of an optimal concentration is crucial in reducing acquisition times, and thus in ensuring clinical feasibility. It was shown that to obtain a SR of $\lambda / 10$ while imaging the slow moving microvasculature flow at $5 \mathrm{~cm}$ depth, the acquisition time could be as low as 56 seconds. If SonoVue ${ }^{\mathrm{TM}}$ microbubbles are administered as an intravenous infusion (VueJect ${ }^{\mathrm{TM}}$, Bracco, Milan) at a rate of $5 \mathrm{ml} / \mathrm{min}$, it is estimated that with an average of 300 million microbubbles per $\mathrm{ml}$ [31], and an average human blood volume of 4.9 litres, 5102 microbubbles will be introduced per millilitre per second. After 30 seconds, $1.5 \times 10^{5}$ bubbles/ $\mathrm{ml}$ will have been introduced, compared to an optimum signal density provided by the model of approximately $0.1-1.5 \times 10^{5}$ signals $/ \mathrm{ml}$. Accounting for a reduction in the number of microbubbles which are detected during imaging due to issues such as dissolution, the proportion of bubbles reaching the target area, and the polydispersed nature of the microbubble population, current estimations of signal density appear to be in the practical range for clinical imaging.

'Dencks et al 2017 provided an exponential expression for the acquisition time needed for the localisation coverage in an image to saturate to a value assumed to be proportional to relative blood volume (rBV). The reliability of rBV estimates from shortened measurement times are then examined experimentally for a specific imaging target and imaging parameters (bolus scans of mouse tumours). Our study instead aims to develop a generalised model which is able to predict the required imaging time for US-SR from user-input imaging parameters, microbubble concentrations and target vasculature. The model aims to predict the optimal microbubble signal density for specific imaging conditions for US-SR. In order to test the example studied in Dencks et al 2017 using our model, imaging parameters and tissue targets comparable to those in the 2017 study (40 MHz transmit frequency, $5 \mu \mathrm{m}$ resolution, $50 \mathrm{~Hz}$ frame rate, bolus injection) are used. The estimated acquisition time to obtain $90 \%$ of the localizations required to cover vascular regions using our model was comparable to that to obtain $90 \%$ of the final vascular coverage (between 78-139 seconds depending on the peak blood concentration during bolus injection, and 50-101 seconds respectively).'

Signal density, the density of bubble signals detected in the acquisition, will vary depending on many details of the imaging acquisition, including transmit frequency, bubble population and behavior, bandwidth, and background noise. The corresponding suitable injection concentration will depend not only on these factors, but also aspects of the practical set-up, e.g. the proportion of bubbles reaching the target area, the disease condition, and administration type (bolus/infusion), so could be patient and disease dependent.

Indeed, an increase in temporal resolution using fast plane waves should provide a higher bubble localization rate for a given microbubble concentration (if bubbles are not destroyed), and moreover, may improve velocity estimations due to more frequent sampling. Since SR imaging relies upon the combined contributions of many localizations over time, for a given microbubble concentration, a greater frame rate should therefore result in a decrease in the overall acquisition time. This, however, does not take into account flow, and therefore this justification is more relevant to situations in which individual signals are activated and deactivated. Nevertheless, the SR technique requires that the microbubbles sample the entire microvascular structure during acquisition to provide full spatial information. This, therefore, places a limit on the minimum imaging time possible for adequate visualization.

In the case of fast imaging of moving bubbles, two competing factors are at play when thinking about acquisition time; these relate to blood flow velocity and frame rate. Firstly, in order for microbubbles to provide new spatial information in each frame, the 
bubbles must be moving, and their position in each frame should contribute additional spatial information to the final rendering. As such, one can define 'supplementary' information to be the occurrence of a bubble localization in frame $n+1$ in which the bubble has moved beyond the localization precision for the same bubble in frame $n$.

Conversely, the frame rate should be high enough that bubble motion during multi-pulse frames does not drastically affect the result of coherent compounding. The compounding of image data involves sending multiple plane wave transmissions for each nonlinear plane wave pulse, i.e. for each phase inverted or amplitude modulated pulse in PI, AM, or PIAM transmission. Bubble movement between each plane wave transmission may mean the plane waves may not be added coherently, and could result in artefacts, incomplete suppression of linear targets, or a smearing or spreading of nonlinear signals in the direction of motion. It is noted that an axial displacement of approximately half a pulse wavelength during the time required to acquire a frame will lead to destructive interference in the compounding operation and as a result cause image degradation [32]. Higher phase coherence is required to avoid motion artefacts in the axial direction than the lateral since the spatial frequency in the axial direction is much higher, while in the lateral direction the point spread function acts as a spatial low-pass filter [32], [33]. Smearing due to fast bubble movement in compounded images may cause a reduction in localization accuracy. Nevertheless, this should not cause a problem to the final visualization if the bubble trajectory remains within the lumen of the vessel, i.e. without sharp change in direction during each pulse sequence.

Results demonstrated that the optimum probability of detecting isolated bubbles occurs over a range of tissue types throughout bolus circulation. While this means that the optimal signal density for an object of interest is not constant, it provides the opportunity to obtain SR images over a range of tissue types. These results also demonstrate that there will be preferential times post-injection to visualize certain tissue types.

Even with a constant microbubble signal density, the considerable variation in tissue blood volume fraction means that regions in the image may have a considerably lower probability of detecting isolated bubbles and therefore parts of image may not be fully represented.

Furthermore, the probability of imaging isolated microbubbles within the diffraction limit varies with time after a bolus injection, where the optimum probability will be in areas of high tissue blood volume immediately after contrast arrival. After fast inflow, the peak probability will change to lower tissue blood volumes, and will slowly change back to higher blood volumes as the contrast agent dissolves and is no longer remains in the blood stream.

There are a number of assumptions implemented in this model which could lead to discrepancies between modelled and experimental findings. The model assumes an ideal SR algorithm which is able to correctly detect single bubbles in all cases, and reject those from multiple bubbles within one PSF sized volume. A more realistic imaging scenario would incorporate noise, as well as varying bubble signals such as ringing or interference signals created by clouds of bubbles; these would affect the ability of the algorithm to identify and accurately localize bubbles. A condition to account for the uneven spatial distribution of events could also be added in future models [34]. Additionally, in general, $V_{P S F}$ is not isotropic; the elevational resolution is typically much larger than in-plane resolutions in $2 \mathrm{D}$, and this can be readily modelled by the method presented here. Three-dimensional results can be easily extrapolated from the model by setting a diffraction limited resolution and localization precision measure in the elevational direction, $\mathrm{FWHM}_{z}$ and $\sigma_{z}$ respectively.

Acquisition times longer than 10 minutes would currently be undesirable in a clinical setting due to motion effects and the use of both clinician and patient time. Acquiring data with a $3 \mathrm{D}$ probe is vital 
for future clinical application. Modelling of US-SR in a $3 \mathrm{D}$ setting is required to explore the parameters required to minimize this time.

The standard clinical dose of SonoVue microbubbles in bolus form has been shown in clinical experiments to be far higher than desired for US-SR at its peak concentration. Instead, a slow infusion of the same clinical dose is preferred due to its lower signal density, and longer potential imaging time due to replenishment. The calculation of the required microbubble concentration prior to imaging based on the imaging sample volume is challenging, and is likely to be unfeasible in a clinical environment. Sustaining a suitable concentration of microbubble scatterers within the image volume may instead require development of an automatic feedback system that regulates the bubble concentration. By automatically monitoring the bubble density per frame during image acquisition according to the optimum predicted by Poisson statistics, the concentration information could be used to drive an infusion pump delivering adjustable microbubble infusion rates. This work could therefore form a basis for the development of more complex and realistic models for SR in the future. One possible approach for overcoming this limitation is high-density imaging. By increasing the density potential localizations per frame, shorter acquisition times could be achieved. High density methods which exist for optical microscopy, such as DAOSTORM, which fits multiple overlapping PSFs in an iterative manner by analyzing pixel clusters in the residual image and obtains localizations by minimizing a leastsquares criterion, and CS-STORM (Compressed sensing STORM), which imposes sparsity priors on the distribution of signal sources and localizes based on a convex optimization problem, can provide increased recall rates. Various sparsitybased techniques have recently been adopted in the ultrasound field [16], [35] [36] to reduce acquisition times.
The number of required frames has been shown to increase for a decrease in transmit frequency and for an increase in SR precision, so will increase the acquisition time for any given frame rate.

\section{CONClusion}

Calculations based on Poisson statistics demonstrate the importance of retaining an appropriate microbubble signal density to maintain viable acquisition times for clinical implementations. Too high and the occurrence of multiple, inseparable signals will limit the number of isolated signals detected, too low and the requirement for a large number of frames will mean a long acquisition time. This, along with the image acquisition procedure, transmit frequency, localization precision, and desired super-resolved image signal to noise ratio together determine the optimal acquisition time for SR imaging for a fixed flow velocity. Exploring the effects of both system parameters and details of the target vasculature can allow better choice of acquisition settings, and provide improved understanding of the completeness of SR information.

\section{REFERENCES}

[1] N. Weidner and J. Folkman, "Tumoral vascularity as a prognostic factor in cancer.," Important Adv. Oncol., pp. 167-90, Jan. 1996.

[2] N. Weidner, J. Folkman, F. Pozza, P. Bevilacqua, E. N. Allred, D. H. Moore, S. Meli, and G. Gasparini,

"Tumor angiogenesis: a new significant and independent prognostic indicator in early-stage breast carcinoma.," J. Natl. Cancer Inst., vol. 84, no. 24, pp. 1875-87, Dec. 1992.

[3] R. F. Tuma, W. N. Duran, and K. Ley, Microcirculation. Academic Press, 2011.

[4] American Diabetes Association, "Peripheral Arterial Disease in People with Diabetes," Diabetes Care, vol. 26, no. 12, pp. 3333-3341, 2003.

[5] M. J. Fowler, "Microvascular and Macrovascular Complications of Diabetes," Clin. Diabetes, vol. 26, no. 2, pp. 77-82, Apr. 2008.

[6] P. E. Norman, J. W. Eikelboom, and G. J. Hankey, "Peripheral arterial disease: prognostic significance and prevention of atherothrombotic complications.," Med. J. Aust., vol. 181, no. 3, pp. 150-4, 2004.

[7] O. M. Viessmann, R. J. Eckersley, K. ChristensenJeffries, M. X. Tang, and C. Dunsby, "Acoustic superresolution with ultrasound and microbubbles.," Phys. 
Med. Biol., vol. 58, no. 18, pp. 6447-58, Sep. 2013. K. Christensen-Jeffries, R. J. Browning, M.-X. Tang, C. Dunsby, and R. J. Eckersley, "In Vivo Acoustic Super-Resolution and Super-Resolved Velocity Mapping Using Microbubbles," IEEE Trans. Med. Imaging, vol. 34, no. 2, pp. 433-440, 2015.

[9] M. A. O'Reilly and K. Hynynen, "A super-resolution ultrasound method for brain vascular mapping.," Med. Phys., vol. 40, no. 11, p. 110701, Nov. 2013.

[10] D. Ackermann and G. Schmitz, "Reconstruction of flow velocity inside vessels by tracking single microbubbles with an MCMC data association algorithm," IEEE Int. Ultrason. Symp. IUS, pp. 627630, 2013.

[11] C. Errico, J. Pierre, S. Pezet, Y. Desailly, Z. Lenkei, O. Couture, and M. Tanter, "Ultrafast ultrasound localization microscopy for deep super-resolution vascular imaging," Nature, vol. 527, no. 7579, pp. 499-502, Nov. 2015.

[12] F. Lin, S. E. Shelton, D. Espíndola, J. D. Rojas, G. Pinton, and P. A. Dayton, "3-D Ultrasound Localization Microscopy for Identifying Microvascular Morphology Features of Tumor Angiogenesis at a Resolution Beyond the Diffraction Limit of Conventional Ultrasound," Theranostics, vol. 7, no. 1, pp. 196-204, 2017.

[13] Y. Desailly, O. Couture, M. Fink, and M. Tanter, "Sono-activated ultrasound localization microscopy," Appl. Phys. Lett., vol. 103, no. 17, 2013.

[14] P. Song, J. D. Trzasko, A. Manduca, R. Huang, R. Kadirvel, D. F. Kallmes, and S. Chen, "Improved Super-Resolution Ultrasound Microvessel Imaging With Spatiotemporal Nonlocal Means Filtering and Bipartite Graph-Based Microbubble Tracking," IEEE Trans. Ultrason. Ferroelectr. Freq. Control, vol. 65, no. 2, pp. 149-167, Feb. 2018.

[15] T. Opacic, S. Dencks, B. Theek, M. Piepenbrock, D. Ackermann, A. Rix, T. Lammers, E. Stickeler, S. Delorme, G. Schmitz, and F. Kiessling, "Motion model ultrasound localization microscopy for preclinical and clinical multiparametric tumor characterization," Nat. Commun., vol. 9, no. 1, pp. 113, 2018.

[16] O. Solomon, R. J. G. van Sloun, H. Wijkstra, M. Mischi, and Y. C. Eldar, "Exploiting flow dynamics for super-resolution in contrast-enhanced ultrasound," Apr. 2018.

[17] D. Cosgrove and N. Lassau, "Imaging of perfusion using ultrasound.," Eur. J. Nucl. Med. Mol. Imaging, vol. 37 Suppl 1, pp. S65-85, Aug. 2010.

[18] H. Becher and P. N. Burns, Handbook of contrast echocardiography: left ventricular function and myocardial perfusion. Springer, 2000.

[19] M. A. Pysz, K. Foygel, C. M. Panje, A. Needles, L. Tian, and J. K. Willmann, "Assessment and monitoring tumor vascularity with contrast-enhanced ultrasound maximum intensity persistence imaging.," Invest. Radiol., vol. 46, no. 3, pp. 187-95, Mar. 2011.

[20] D. Ackermann and G. Schmitz, "Reconstruction of flow velocity inside vessels by tracking single microbubbles with an MCMC data association algorithm," in 2013 IEEE International Ultrasonics Symposium (IUS), 2013, pp. 627-630.

[21] G. P. Luke, A. S. Hannah, and S. Y. Emelianov, "Super-Resolution Ultrasound Imaging in Vivo with Transient Laser-Activated Nanodroplets," Nano Lett., vol. 16, no. 4, pp. 2556-2559, Apr. 2016.

[22] G. Zhang, S. Harput, S. Lin, K. Christensen-Jeffries, C. H. Leow, J. Brown, C. Dunsby, R. J. Eckersley, and M.-X. Tang, "Acoustic wave sparsely activated localization microscopy (AWSALM): Superresolution ultrasound imaging using acoustic activation and deactivation of nanodroplets," Appl. Phys. Lett., vol. 113, no. 1, p. 014101, Jul. 2018.

[23] K. Christensen-Jeffries, J. Brown, P. Aljabar, M. Tang, C. Dunsby, and R. J. Eckersley, “3-D In Vitro Acoustic Super-Resolution and Super-Resolved Velocity Mapping Using Microbubbles," IEEE Trans. Ultrason. Ferroelectr. Freq. Control, vol. 64, no. 10, pp. 1478-1486, Oct. 2017.

[24] M. Tanter and M. Fink, "Ultrafast imaging in biomedical ultrasound," IEEE Trans. Ultrason. Ferroelectr. Freq. Control, vol. 61, no. 1, pp. 102 119, Jan. 2014.

[25] R. D. Yates and D. J. Goodman, "Probability and Stochastic Processes: A Friendly Introduction for Electrical and Computer Engineers," p. 496, 2014. F. A. Haight, "A Handbook of the Poisson Distribution," J. R. Stat. Soc. Ser. A, vol. 18, no. 4, Jan. 1967.

[27] M. Siepmann, M. Reinhardt, and G. Schmitz, “A Statistical Model for the Quantification of Microbubbles in Destructive Imaging," Invest. Radiol., vol. 45, no. 10, pp. 592-599, Oct. 2010.

[28] P. N. Burns, S. R. Wilson, and D. H. Simpson, "Pulse inversion imaging of liver blood flow: improved method for characterizing focal masses with microbubble contrast.," Invest. Radiol., vol. 35, no. 1, pp. 58-71, Jan. 2000.

[29] P. J. Phillips, "Contrast pulse sequences (CPS): imaging nonlinear microbubbles," in 2001 IEEE Ultrasonics Symposium. Proceedings. An International Symposium (Cat. No.01CH37263), 2001, vol. 2, pp. 1739-1745.

[30] H. C. Schwickert, T. P. L. Roberts, D. M. Shames, C. F. V. Dijke, A. Disston, A. Mühler, J. S. Mann, and R. C. Brasch, "Quantification of liver blood volume: comparison of ultra short ti inversion recovery echo planar imaging (ulstir-epi), with dynamic 3d-gradient recalled echo imaging," Magn. Reson. Med., vol. 34, no. 6, pp. 845-852, 1995.

[31] M. Schneider, "Characteristics of SonoVue ${ }^{\mathrm{TM}}$," Echocardiography, vol. 16, no. s1, pp. 743-746, Oct. 1999.

[32] J. Wang and J. Lu, "Motion artifacts of extended high frame rate imaging.," IEEE Trans. Ultrason.

Ferroelectr. Freq. Control, vol. 54, no. 7, pp. 13031315, 2007.

[33] B. Denarie, T. A. Tangen, I. K. Ekroll, N. Rolim, H. Torp, T. Bjåstad, and L. Lovstakken, "Coherent plane 
wave compounding for very high frame rate

ultrasonography of rapidly moving targets.," IEEE

Trans. Med. Imaging, vol. 32, no. 7, pp. 1265-76, Jul. 2013.

[34] S. van de Linde, S. Wolter, M. Heilemann, and M. Sauer, "The effect of photoswitching kinetics and labeling densities on super-resolution fluorescence imaging," J. Biotechnol., vol. 149, no. 4, pp. 260-266, Sep. 2010.

[35] A. Bar-Zion, O. Solomon, C. Tremblay-Darveau, D. Adam, and Y. C. Eldar, "Sparsity-based Ultrasound Super-resolution Hemodynamic Imaging,” Dec. 2017.

[36] R. J. G. van Sloun, O. Solomon, Y. C. Eldar, H. Wijkstra, and M. Mischi, "Sparsity-driven superresolution in clinical contrast-enhanced ultrasound," in 2017 IEEE International Ultrasonics Symposium (IUS), 2017, pp. 1-4.

[37] Center for drug evaluation and research application number: 203684orig1s000 clinical pharmacology and biopharmaceutics review(s), "SonoVue: clinical

pharmacology and biopharmaceutics review(s)." 2013. 